\title{
Usos Finais de Eletricidade e Rotinas de Uso como Base para Estratégias de Eficiência Energética por Meio de Auditoria Residencial
}

\author{
SILVA, Arthur Santos ${ }^{1}$ \\ LUIZ, Fernando \\ MANSUR, Ana Carolina \\ GHISI, Enedir \\ ${ }^{1}$ Universidade Federal de Santa Catarina, Florianópolis, Brasil. arthurssilva07@gmail.com
}

\section{Resumo}

O trabalho tem por objetivo apresentar os usos finais de eletricidade e rotinas de uso em habitações de interesse social. Os dados foram obtidos por meio de auditoria energética em uma amostra de 60 habitações de interesse social da região de Florianópolis-SC, por meio de aplicação de questionários aos moradores e medições do consumo de energia elétrica. Os dados obtidos com os questionários se referem à situação socioeconômica dos moradores e às rotinas de uso dos equipamentos eletroeletrônicos e iluminação, estimados para períodos de verão e inverno. As medições foram utilizadas para a caracterização dos equipamentos e lâmpadas, determinação do consumo de energia elétrica e o tempo de uso, por um período maior do que duas semanas em cada habitação. O tratamento de dados foi feito em forma de intervalos de confiança, por meio de métodos estatísticos paramétricos e não paramétricos. Os resultados mostraram que o chuveiro elétrico representa o maior uso final da amostra, com 36,8\% de mediana, seguido dos refrigeradores, televisão e iluminação. Os dados não mostraram diferenças significativas entre os períodos de verão e inverno, devido ao tamanho reduzido da amostra e à variabilidade dos dados. As rotinas de uso encontradas, quando associadas à densidade potência média respectiva, são variáveis de entrada para estudos de simulação computacional do desempenho térmico dessas habitações, além de ajudarem na caracterização desse tipo de edificação. A correta quantificação dos usos finais de eletricidade de amostras pequenas leva à adoção de medidas corretas e específicas para a eficiência energética dessas habitações.

Palavras-Chave: auditoria energética, usos finais de eletricidade, habitação de interesse social.

\begin{abstract}
The paper aims to present the electricity end-uses and schedules for occupancy and equipment usagefor low-income houses. Data were obtained through energy audit on a sample of 60 low-income houses in the region of Florianópolis-SC, by means of questionnaires and measurements of electricity consumption. The data obtained from the questionnaires refer to the socioeconomic status of the householders and the schedules of equipment and lighting usage, estimated for summer and winter periods. The measurements were used to characterize the equipment and lamps, determining the electricity consumption and usage time, for a period longer than two weeks in each house. The data were assessed through confidence intervals, with parametric and nonparametric statistics. The results showed that the electric shower represents the largest end-use of the sample, with a median of $36.8 \%$, followed by refrigerators, television and lighting. The data showed no significant differences between summer and winter periods, due to the small sample size and the variability of the data. The usage schedules found, when related with respective average power density, correspond to input variables for building simulation studies on the thermal performance of these houses, and contribute to characterize this type of building. The correct quantification of electricity end-uses from small samples leads to the adoption of specific measuresfor energy efficiency in these houses.
\end{abstract}

Keywords: energy audits, electricity end-uses, low-income houses. 


\section{Introdução}

O crescimento econômico de um país está associado ao crescimento do consumo de energia e juntamente com demais impactos associados, tendo em vista principalmente a mudança do estilo de vida das pessoas (CHUNG-SHENG; SHU-WEN; XIN, 2012).

No caso do Brasil, o consumo de eletricidade cresceu aproximadamente 56\% no período de 2002 a 2011. Atualmente, o setor residencial representa $23,6 \%$ do consumo total de energia elétrica do país (BRASIL, 2012a).

O consumo de energia em habitações depende de diversos fatores e atividades dos ocupantes, que são relacionadas com os usos finais de eletricidade (BIN; DOWLATABADI, 2005). Importantes estudos foram realizados no sentido de determinar os usos finais de eletricidade no setor residencial brasileiro (e. g. JANNUZZI; SCHIPPER, 1991; ALMEIDA; SCHAEFFER; LA ROVERE, 2001; GHISI; GOSCH; LAMBERTS, 2007) que ajudam a caracterizar o perfil de consumo e auxiliam no desenvolvimento de estratégias para a eficiência energética.

A determinação dos usos finais de eletricidade é uma etapa primordial para o desenvolvimento de estratégias de eficiência energética. Por meio dela, pode-se identificar que tipo de uso prepondera no consumo final de eletricidade possibilitando maior foco no desenvolvimento de novas tecnologias e eficiência energética.

Santana e Hayashi (1997) fizeram um trabalho sobre o diagnóstico energético de residências em Salvador, BA. Foi verificado que a eletricidade representa o maior consumo energético, em frente ao gás liquefeito de petróleo (GLP),e a maior parte do consumo de eletricidade é devido aos refrigeradores. O chuveiro elétrico mostrou uso final praticamente constante em todas as faixas de renda analisadas. Foi verificada a correlação direta entre o consumo de energia e a renda familiar.

Ghisi, Westphal e Lamberts (1997) apresentam resultados de potências médias de diversos equipamentos residenciais a partir de medições de consumo de energia e tempo de uso. Os resultados demonstram que a determinação das potências médias leva à estimativa de usos finais mais precisos do que a utilização de potências nominais.

Fedrigo, Ghisi e Lamberts (2009) fizeram estimativas dos usos finais de eletricidade em edificações residenciais para cinco regiões geográficas brasileiras. Os dados foram obtidos pelo Programa Nacional de Conservação de Energia Elétrica da Eletrobrás (PROCEL). A análise demonstrou que as variáveis de clima, renda familiar e aspectos culturais influenciam diretamente no consumo de energia, principalmente no caso do chuveiro elétrico.

De acordo com Druckman e Jackson (2009), um aumento do nível socioeconômico direciona para uma expansão do consumo de energia, devido a uma consequência da melhoria do conforto e lazer das pessoas.

Sobre o nível socioeconômico, Ghisi, Gosch e Lamberts (2007) também verificaram que a renda está diretamente associada com o consumo de energia. O aumento do salário mínimo de $\mathrm{R} \$ 200,00$ para $\mathrm{R} \$ 622,00$ na última década (BRASIL, 2007; 2011) pode ter contribuído para um aumento no consumo de energia nesse período.

Nesse contexto, o Brasil desenvolve diversos programas de eficiência energética em edificações residenciais, como o PROCEL Edifica e o Programa Brasileiro de Etiquetagem, que tratam da edificação em si e também da eficiência de cada equipamento elétrico residencial individualmente. Em todos eles, é imprescindível a correta quantificação dos usos finais de eletricidade e das rotinas de uso, para que o programa em questão seja eficaz.

\section{Objetivo}

O objetivo deste trabalho é apresentar os usos finais de eletricidade e rotinas de uso de equipamentos obtidos através de auditoria energética em habitações de interesse social de Florianópolis-SC.

\section{Método}

O método é dividido em coleta de dados do consumo de eletricidade, tratamento de dados e análise de dados.

\subsection{Coleta de dados}

Os dados foram obtidos através de auditoria energética residencial com equipamentos de medição e monitoramento do consumo de eletricidade, e pesquisa estruturada por meio de questionários. As pesquisas foram feitas em períodos de verão e inverno do ano de 2012.

\subsubsection{Amostra}

Foram selecionadas aleatoriamente 60 habitações de interesse social de Florianópolis-SC. As habitações foram classificadas pela sua localização em áreas de baixa renda com base no Plano Municipal de 
Habitações de Interesse Social (PMF, 2012).

As habitações possuem área construída de 25 a 85 $\mathrm{m}^{2}$, de 1 a 8 moradores, de acordo com a Figura 1 . 0 sistema construtivo predominantefoi alvenaria de tijolos cerâmicos, e algumas habitações de madeira.

Figura 1: Área construída e número de moradores da amostra de habitações.
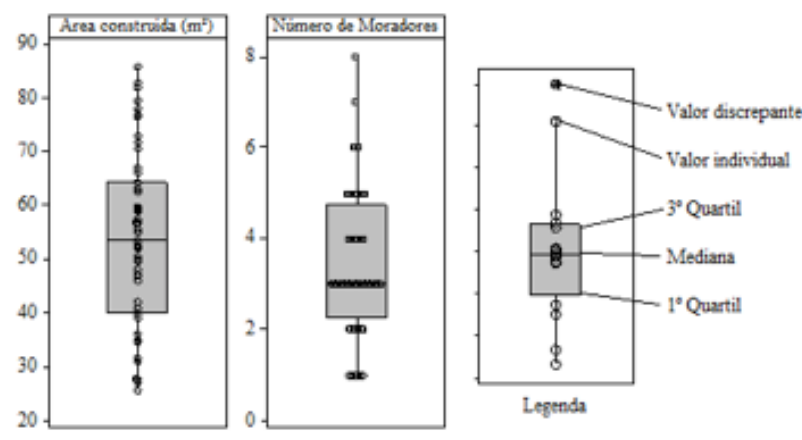

\subsubsection{Escopos e questionários}

Foram utilizados três tipos de questionários durante as entrevistas: (1) socioeconômico, (2) usos finais de eletricidade e (3) rotinas de uso dos equipamentos eletroeletrônicos.

O questionário socioeconômico reuniu informações a respeito da renda de cada morador, a renda total familiar e o número de moradores.

No questionário de usos finais de eletricidade, todos os equipamentos eletroeletrônicos da edificação foram registrados por meio das seguintes características: tipo, modelo, consumo de eletricidade, e em qual cômodo se localiza. Também foram obtidas as faturas de eletricidade da concessionária local dos últimos 12 meses, para análise de validação das estimativas.

O questionário de rotinas de uso dos equipamentos reuniu informações sobre o tempo de utilização dos mesmos, em cada horário do dia, e a frequência de ocorrência no mês. Essas rotinas de uso foram estimadas pelos moradores, para períodos de verão e inverno, obtendo-se dados de fração horária para cada evento.

\subsubsection{Equipamentos de medição}

O consumo de eletricidade de cada equipamento eletroeletrônico da habitação foi medido e monitorado por um período mínimo de duas semanas em cada habitação. Para isso, dois tipos de medidores foram utilizados: (1) PowerBall T8 e (2) CEM 1000.

O medidor PowerBall T8 foi utilizado para determinar o tempo total de uso do equipamento e a eletricidade total consumida no período. O medidor é limitado a equipamentos de potência menor ou igual a $2,2 \mathrm{~kW}$, o que inclui: geladeira, refrigeradores, máquina de lavar roupa, micro-ondas, televisão, computadores, ferro de passar roupa, ventiladores, cafeteira, secador de cabelos, entre outros. O chuveiro elétrico foi caracterizado pela potência nominal fornecida pelo fabricante, sendo que nenhum chuveiro encontrado foi do tipo de potência variável.

Desta forma, foram obtidas informações de consumo de eletricidade e tempo total de uso de cada equipamento, em cada uma das habitações, possibilitando o cálculo da potência média dos equipamentos.

O medidor CEM 1000 foi utilizado para medir as características das lâmpadas, como: potência instantânea, fator de potência, tensão e corrente. As lâmpadas não foram monitoradas para a determinação da potência média, mas apenas da potência instantânea.

\subsection{Tratamento de dados}

O tratamento de dados foi realizado para a determinação de valores representativos dos usos finais de eletricidade e da determinação de intervalos de confiança para as rotinas de uso e os usos finais.

\subsubsection{Rotinas de uso dos equipamentos}

Os dados obtidos foram representados de forma que, em cada uma das 24 horas do dia (para o verão e para o inverno) houvesse o tempo de uso de cada equipamento em minutos.

A potência média dos equipamentos eletroeletrônicos foi obtida por meio da Equação 1.

$$
\mathrm{PM}_{\mathrm{i}}=\frac{\mathrm{CE}_{\mathrm{i}}}{\mathrm{T}_{\mathrm{i}}}
$$

Onde:

$\mathrm{PM}_{\mathrm{i}} \quad$ é a potência média do equipamento i em cada habitação (W);

$\mathrm{CE}_{\mathrm{i}}$ é o consumo de eletricidade do equipamento i em cada habitação $(\mathrm{Wh})$;

$T_{i} \quad$ é o tempo total de uso do equipamento i em cada habitação $(h)$.

Uma das aplicações das rotinas de uso é na simulação termoenergética de edificações, onde são consideradas as rotinas de uso de equipamentos e iluminação por meio de frações horárias de picos de carga. Desta forma, para a determinação de rotinas de uso representativas para a utilização nesse tipo de 
programa, os equipamentos foram agrupados em cada ambiente de permanência prolongada e uma rotina de uso média foi calculada para cada ambiente, de acordo com a Equação 2, sendo que a fração de potência em cada ambiente é calculada conforme a Equação 3.

$$
\mathrm{PMH}_{\mathrm{i}}=\sum_{\mathrm{j}=0}^{\mathrm{n}} \frac{\mathrm{PM}_{\mathrm{ij}}}{\mathrm{n}}
$$

\section{Onde:}

$\mathrm{PMH}_{\mathrm{i}}$ é a potência média do equipamento i em todas as habitações (W);

$\mathrm{PM}_{\mathrm{ij}}$ é a potência média do equipamento $\mathrm{i}$ em cada habitação j $(W)$;

n é o número total de habitações (adimensional).

$$
\mathrm{FP}_{\mathrm{j}}=\frac{\sum_{\mathrm{i}=0}^{\mathrm{m}} \mathrm{FP}_{\mathrm{ij}} \times \mathrm{PMH}_{\mathrm{i}} \times \mathrm{n}_{\mathrm{i}}}{\mathrm{n} \times \sum_{\mathrm{i}=0}^{\mathrm{m}} \mathrm{PMH}_{\mathrm{i}}}
$$

Onde:

$\mathrm{FP}_{\mathrm{j}} \quad$ é a fração de potência em cada horário j do dia para cada ambiente de permanência prolongada (adimensional);

j é o horário do dia, variando de 0 a 23 (horas);

$\mathrm{FP}_{\mathrm{ij}} \quad$ é a fração de potência do equipamento i no horário $\mathrm{j}$ do dia, em cada ambiente de permanência prolongada (adimensional);

m é o número de equipamentos (adimensional);

$\mathrm{n}_{\mathrm{i}} \quad$ é o número de habitações que contém o equipamento i (adimensional);

n é o número total de habitações (adimensional);

$\mathrm{PMH}_{\mathrm{i}}$ é a potência média do equipamento i nas habitações (W).

Finalmente, cada valor de $\mathrm{FP}_{\mathrm{j}}$ de 0 a 23 horas representa uma rotina de uso representativa para cada ambiente de permanência prolongada da edificação, associado à soma das potências médias dos equipamentos em cada ambiente.

\subsubsection{Usos finais de eletricidade}

O consumo total diário de eletricidade com cada equipamento foi calculado por meio da Equação4.

Onde:

$$
\mathrm{CEd}_{\mathrm{i}}=\sum_{0}^{24} \mathrm{PM}_{\mathrm{i}} \times \mathrm{t}_{\mathrm{i}}
$$

$\mathrm{CEd}_{\mathrm{i}}$ é o consumo de eletricidade diário de cada equipamento i em cada habitação (Wh/dia);

$\mathrm{PM}_{\mathrm{i}} \quad$ é a potência média do equipamento i em cada habitação $(\mathrm{W})$; $t_{i}$ é o tempo de uso de fracionado em cada hora do dia, entre 0 e 1 (horas/dia).

O consumo de energia mensal de cada equipamento foi determinado pela multiplicação do consumo diário pelo número de dias que é utilizado em cada mês, conforme a entrevista com o morador.O consumo de energia mensal de cada habitação foi determinado por meio daEquação 5 .

$$
\mathrm{CEm}=\sum_{\mathrm{i}}^{\mathrm{n}} \mathrm{CEd}_{\mathrm{i}} \times \mathrm{d}_{\mathrm{i}}=\sum_{\mathrm{i}}^{\mathrm{n}} \mathrm{CEm}_{\mathrm{i}}
$$

Onde:

CEm é o consumo de energia mensal de cada habitação (Wh/mês);

$\mathrm{n} \quad$ é o número total de equipamentos em cada habitação (adimensional);

$\mathrm{CEd}_{\mathrm{i}}$ é o consumo de energia diário de cada equipamento i em cada habitação (Wh/dia);

$\mathrm{d}_{\mathrm{i}} \quad$ é o número de dias que o equipamento i é utilizado no mês (dias/mês);

$C E m_{i}$ é o consumo de energia mensal de cada equipamento i (Wh/mês).

O uso final de cada equipamento é determinado através da Equação 6, para cada habitação.

$$
\mathrm{UF}_{\mathrm{i}}=100 \frac{\mathrm{CEm}_{\mathrm{i}}}{\mathrm{CEm}}
$$

Onde:

UF $_{\mathrm{i}}$ é o uso final de cada equipamento i em cada habitação (\%);

$\mathrm{CEm}_{\mathrm{i}}$ é o consumo de eletricidade mensal de cada equipamento i em cada habitação (Wh/mês);

CEm é o consumo de eletricidade mensal de cada habitação (Wh/mês).

\subsection{Análise de dados}

Ao todo, três análises foram realizadas: (1) análise de validação do consumo de eletricidade, (2) análise dos usos finais de eletricidade, (3) análise das rotinas de uso dos equipamentos. Todas as análises estatísticas foram realizadas com o programa MiniTab 16.

\subsubsection{Análise de validação do consumo de eletricidade}

O consumo mensal de eletricidade de cada habitação foi comparado com a média mensal dos consumos de eletricidade medidos pela concessionária de energia 
elétrica nos últimos 12 meses. Se a diferença entre o consumo mensal estimado com o consumo médio faturado fosse maior do que $20 \%$, a edificação seriaexcluída das análises. O critério foi definido apenas para os fins deste trabalho, tendo por objetivo evitar a exclusão de muitas habitações da análise e, ao mesmo tempo, manter um nível razoável de precisão.

O processo descrito resultou na exclusão de sete edificações que não atenderam a diferença de $20 \%$.

\subsubsection{Análise dos usos finais}

Os usos finais foram calculados para cada habitação conforme o Item 3.2.2. Para mostrar uma tendência da amostra encontrada, foi realizada a determinação do intervalo de confiança de $90 \%$ para a mediana da amostra com o teste não paramétrico de Wilcoxon, obtendo-se uma generalização da população amostrada.

Os testes não paramétricos são independentes de aderência às distribuições de probabilidades conhecidas e são computados para a mediana da amostra. A única suposição é que a amostra é oriunda de uma distribuição contínua e simétrica em torno da mediana (SIEGEL, 2006).

\subsubsection{Análise das rotinas de uso}

Do item de tratamento de dados, foram obtidas rotinas representativas para cada habitação, considerando a potência média de cada equipamento, a fração de potência em cada horário e a existência do mesmo em cada habitação.

Neste item, as rotinas foram generalizadas para a população por meio de intervalos de confiança de $80 \%$ através do teste não paramétrico de Wilcoxon. A confiabilidade de $80 \%$ foi escolhida devido à grande variabilidade dos dados, e também ao reduzido tamanho da amostra.

Cada valor da variável [FPप_j da Equação 3 entre as habitações foi utilizado na análise.

Este item resulta em rotinas de uso para os equipamentos e iluminação em cada ambiente de permanência prolongada, representando toda a amostra. Para o chuveiro elétrico foi determinada rotina de uso separadamente.

A potência média instalada (em W-Watts) em cada ambiente em cada habitação foi convertida para densidade de potência média $\left(\mathrm{em} \mathrm{W} / \mathrm{m}^{2}\right)$, sendo a potência absoluta de cada equipamento dividida pela área de cada ambiente. A densidade de potência foi generalizada para a amostra por meio do Teste-t de
Student com $80 \%$ de confiabilidade, assumindo-se a normalidade dos dados.

Desta forma, com a conciliação da rotina de uso com a densidade de potência média, encontra-se o consumo de energia mensal que representa a população amostrada.

\section{Resultados}

Este item apresenta os resultados separados por usos finais de eletricidade e rotinas de uso.

\subsection{Usos finais de eletricidade}

A Tabela 1 mostra os usos finais de eletricidade de acordo com o tratamento realizado com o método não paramétrico de $90 \%$ de confiabilidade. Os valores discrepantes foram retirados da análise e a ordem dos usos finais é baseada na mediana da amostra. A categoria "Outros" representa equipamentos que individualmente não contribuíram significativamente para os usos finais e apresentaram grande variabilidade entre as habitações amostradas.

De acordo com Ghisi, Gosch e Lamberts (2007) o consumo de eletricidade com o chuveiro elétrico representa 14 a $28 \%$ dos usos finais no verão e $26 \%$ no inverno, para habitações da Região Sul do Brasil. No mesmo estudo, o refrigerador apresentou de 33 a $34 \%$ dos usos finais no verão e $30 \%$ no inverno.

Conforme a Tabela 1, o chuveiro elétrico, os refrigeradores, a televisão e iluminação representam juntos de $73,8 \%$ a $91,7 \%$ do consumo total de eletricidade das habitações.

Conforme análise de Fedrigo, Ghisi e Lamberts (2009), no verão o chuveiro elétrico representa 39,4\% do consumo total de eletricidade, o refrigerador representa $21,3 \%$ e a televisão $8,7 \%$. No inverno, o chuveiro representa $43,3 \%$, os refrigeradores $20,4 \%$ e a televisão $8,4 \%$. Os resultados são médios, e é verificada a diferença insignificante dos usos finais com refrigeradores e a televisão entre o verão e inverno, mas há certa diferença no uso do chuveiro elétrico.

Neste trabalho, no entanto, os usos finais não mostraram diferença significativa entre os períodos de verão ou inverno, possivelmente devido ao pequeno tamanho da amostra, o que explica a generalização dos usos finais para o ano todo.

Há alguns programas de eficiência energética promovidos por organizações governamentais ou concessionárias de energia. Um exemplo é na região Centro-Oeste, onde a concessionária local efetua 
Tabela 1: Usos finais de eletricidade com intervalo de confiança de $90 \%$ para a mediana.

\begin{tabular}{lccccccc}
\hline Valor & $\begin{array}{c}\text { Chuveiro } \\
\text { elétrico }\end{array}$ & Refrigeradores & Televisão & Iluminação & $\begin{array}{c}\text { Máquina } \\
\text { de lavar } \\
\text { roupas }\end{array}$ & $\begin{array}{c}\text { Micro- } \\
\text { ondas }\end{array}$ & Outros \\
\hline Superior & $40,3 \%$ & $33,1 \%$ & $12,2 \%$ & $6,1 \%$ & $1,1 \%$ & $0,9 \%$ & $13,5 \%$ \\
Mediana & $36,8 \%$ & $29,9 \%$ & $10,2 \%$ & $5,2 \%$ & $0,9 \%$ & $0,6 \%$ & $10,5 \%$ \\
Inferior & $33,5 \%$ & $27,4 \%$ & $8,4 \%$ & $4,5 \%$ & $0,7 \%$ & $0,4 \%$ & $8,0 \%$ \\
\hline
\end{tabular}

doações de refrigeradores mais eficientes, lâmpadas fluorescentes compactas e a adequação do circuito elétrico das habitações. Esse programa beneficiou 32.000 habitações de interesse social até o momento (Brasil, 2012c). As economias estimadas foram de 4.285,41 MWh por ano e redução de 536,48 kW na demanda de pico.

Os resultados do balanço do Procel apontaram redução de 6.604 GWhde consumo de eletricidade em 2011 e $2.619 \mathrm{MW}$ de redução no pico de demanda, sendo a maior redução nos últimos 5 anos (Brasil, 2012b), devido ainiciativas de eficiência energética.

Neste trabalho, o consumo médio mensal de eletricidade foi de $214 \mathrm{kWh}$, e a mediana, $194 \mathrm{kWh}$. De acordo com Ghisi, Gosch e Lamberts (2007), o consumo de eletricidade na Zona Bioclimática onde Florianópolis está localizada é de 7,74 a 8,41 kWh/dia no verão e 8,91 kWh/dia no inverno. Os dados obtidos neste estudo para as 53 habitações apontaram uma média de 7,23 kWh/dia e 7,79 kWh/dia para o verão e inverno, respectivamente.

A Figura 2 mostra a frequência de ocorrência dos dados medidos de consumo de eletricidade por mês (Figura 2-a) e por dia (Figura 2-b) em todas as habitações. Percebe-se que o consumo de eletricidade da amostra teve uma grande amplitude, variando de 80 a $400 \mathrm{kWh} / \mathrm{mês}$, sendo que a maior probabilidade de ocorrência está próxima de 160 kWh/mês.

\subsection{Rotinas de uso dos equipamentos}

A Figura 3 apresenta as rotinas de uso dos equipamentos por ambiente, onde o eixo y representa a fração de potência utilizada, o qual é associada às densidades de potência da Figura 4 para cada ambiente de permanência prolongada.Para o dormitório, por exemplo, a potência média instalada com equipamentos é de $18,28 \mathrm{~W} / \mathrm{m}^{2}$, sendo que a média varia de 10,21 a $26,36 \mathrm{~W} / \mathrm{m}^{2}$, com $80 \%$ de confiabilidade.

Percebe-se que as frações da Figura 3são pequenas em relação às potências médias totais instaladas em cada ambiente, alcançando no máximo fração de 0,33 no dormitório. Essa fração representa de certa forma, um coeficiente de simultaneidade do uso dos equipamentos eletroeletrônicos da edificação.

Conciliando-se a Figura 3 com a Figura 4, pode-se afirmar que, às $20 \mathrm{~h}$, no dormitório, são utilizados de 3,38 a 8,56 Wh/m² (pela multiplicação do intervalo de potências pela respectiva fração de potência) com $80 \%$ de A Figura 5 apresenta as rotinas de uso da iluminação, mostrando a fração de potência e os horários do dia a partir das 17h; nos demais horários a fração é zero. Nesse caso, são apresentados intervalos de confiança de $80 \%$ em nível inferior, mediana e superior. Algumas vezes o nível inferior ou a mediana são nulos, e a barra específica não aparece nas Figuras.Todas as rotinas por ambiente são associadas às densidades de potência mostradas na mesmaFigura 5 , com o teste-t e $80 \%$ de confiabilidade.

Figura 2: Frequência de ocorrência dos consumos de eletricidade na amostraem kWh/mês (a) e em kWh/dia (b).

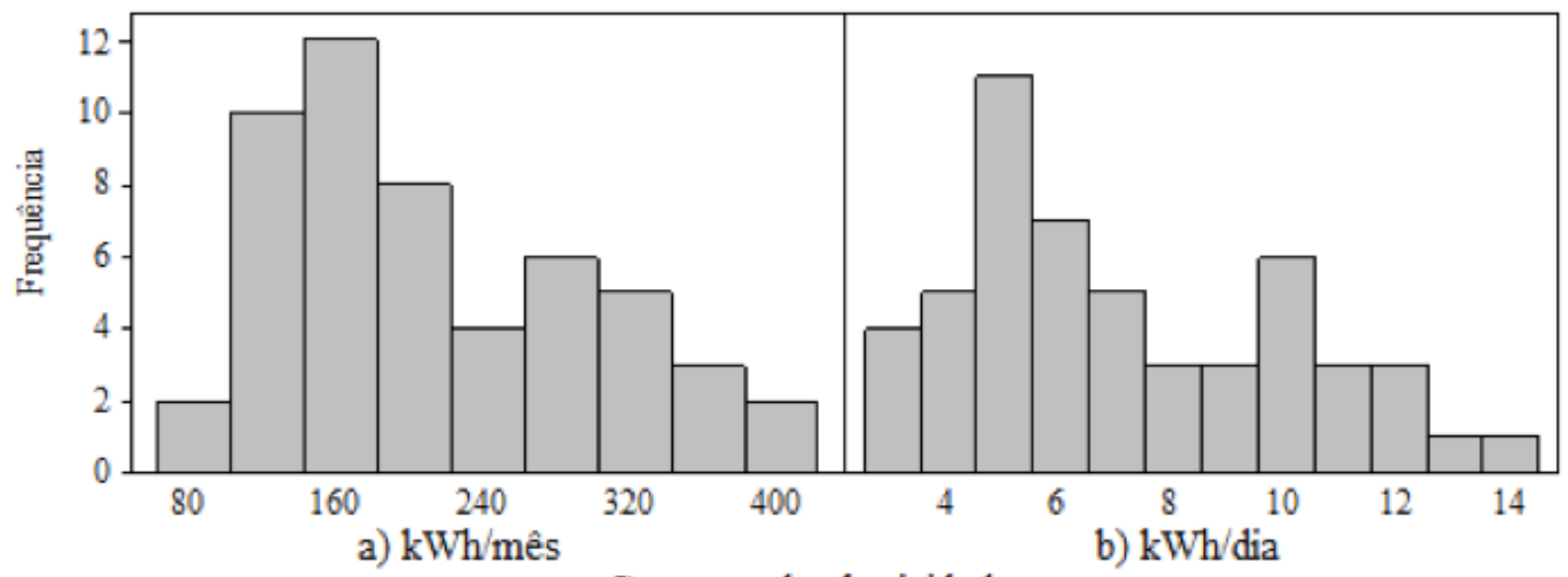

Consumo de eletricidade 
Figura 3: Rotinas médias de uso de equipamentos eletroeletrônicos.

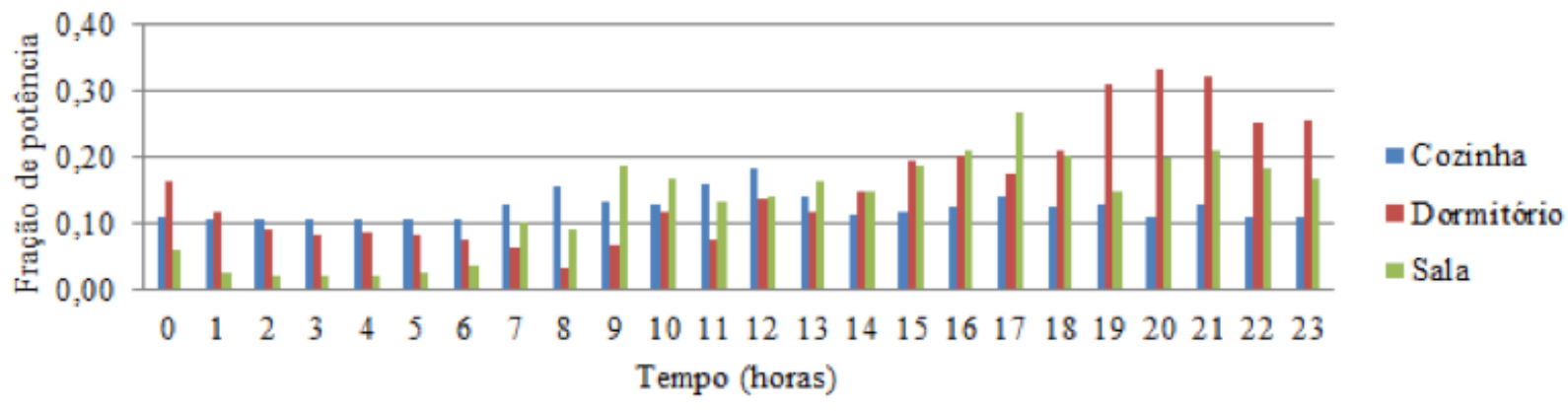

Figura 4: Densidade de potência instalada com equipamentos.

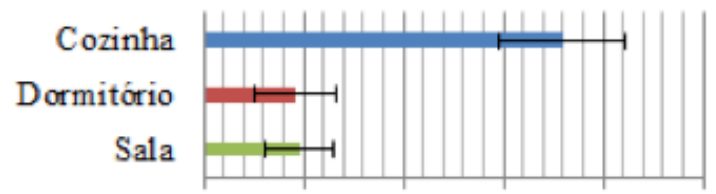

Para o dormitório, por exemplo, a densidade de potência média instalada com iluminação (Figura 5-d) é de $3,82 \mathrm{~W} / \mathrm{m}^{2}$, sendo que a média varia de 3,35 a $4,29 \mathrm{~W} / \mathrm{m}^{2}$, com $80 \%$ de confiabilidade.

Pode-se dizer que, no caso do dormitório, às 20h, a iluminação é utilizada de uma fração de 0,167 a 0,333, que representa de 10 a 20 minutos nessa hora completa. Ao conciliar a fração de potência com a potência média no dormitório, por exemplo, às $20 \mathrm{~h}$,são utilizados de 0,56 a $1,42 \mathrm{Wh} / \mathrm{m}^{2}$, com $80 \%$ de confiabilidade.
Ghisi, Westphal e Lamberts (1997) apresentaram resultados de potências nominais e médias de equipamentos residenciais, por meio de auditoria energética. Foi constatada a necessidade da medição do consumo de energia in loco dos equipamentos, pois a utilização de consumos mensais informados pelo fabricante (para determinadas condições) pode ser bem diferente das condições reais verificadas. No trabalho citado, no caso das geladeiras, o consumo informado pelo fabricantefoi $178 \%$ superior ao medido.

Erros como este podem levar a quantificações incorretas dos usos finais de eletricidade, o que foi contornado pela medição da potência média neste presente estudo.

O chuveiro elétrico, o maior uso final encontrado na amostra desse trabalho, apresenta rotinas de uso e a potência média de acordo com a Figura 6. Os intervalos para as rotinas são de $80 \%$ de confiabilidade

Figura 5: Rotinas de uso de iluminação com intervalos de 80\% de confiabilidade e potências médias, separados por ambiente.

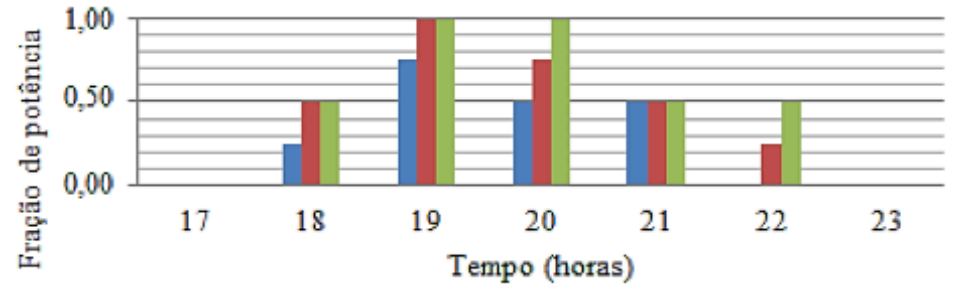

a) rotina de uso da iluminação na cozinha

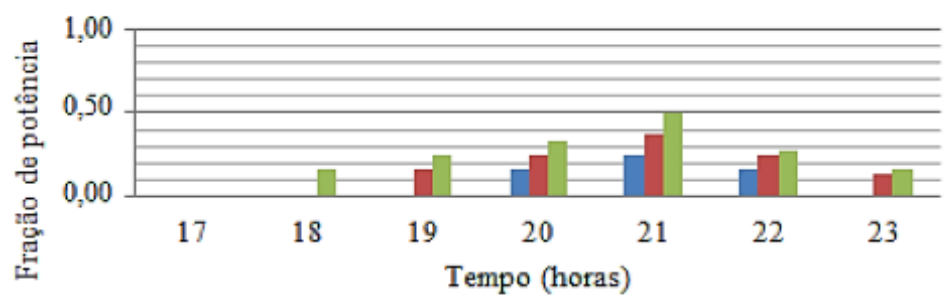

c) rotina de uso da iluminação no dormitório

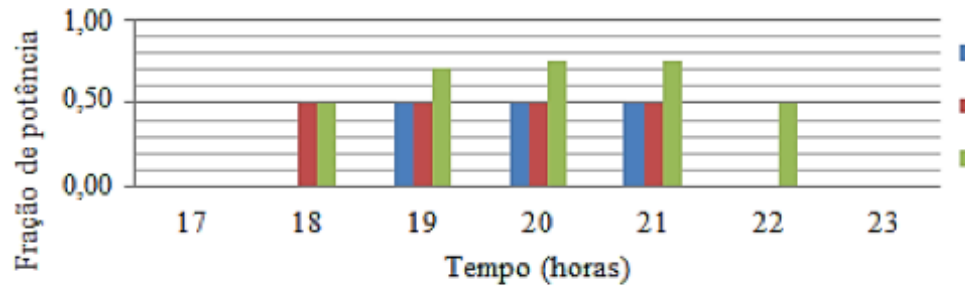

e) rotina de uso da iluminação na sala
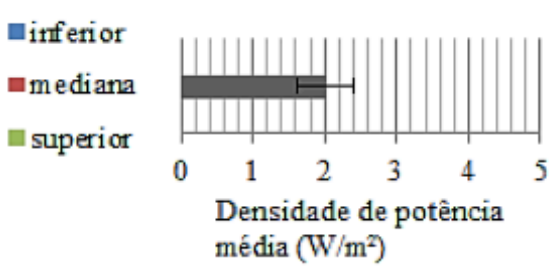

b) potência média de iluminação na cozinha

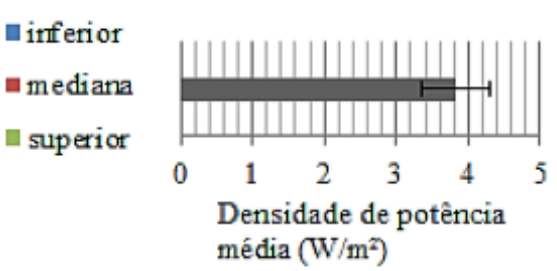

d) potência média de iluminação no dormitório
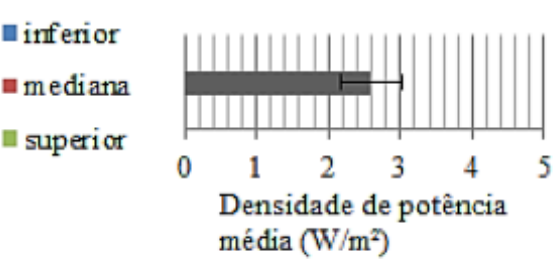

f) potência média de iluminação na sala 
Figura 6: Rotinas de uso e potência média do chuveiro elétrico, com $80 \%$ de confiabilidade.

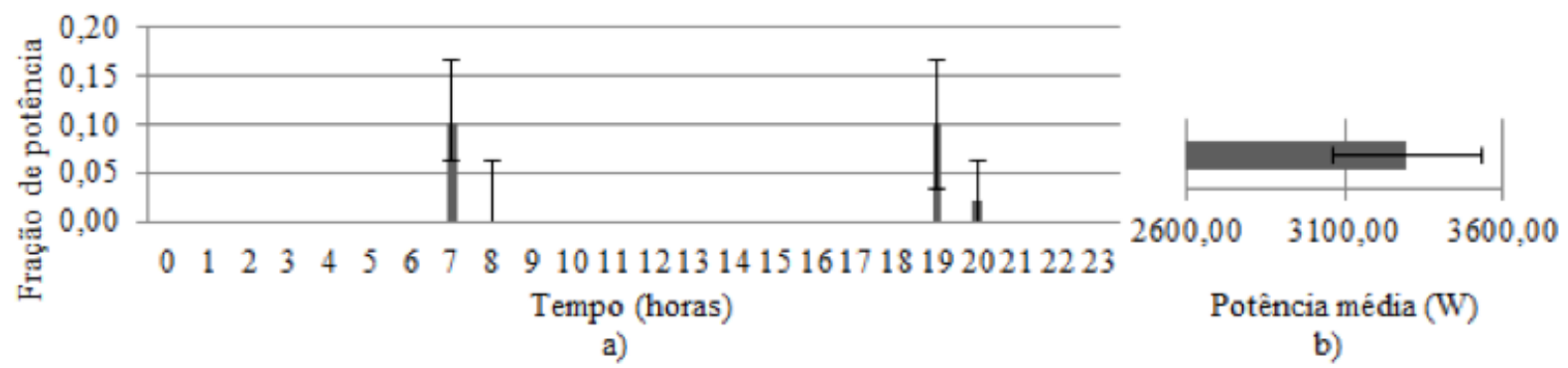

com método não paramétrico, e para a potência, é de $80 \%$ de confiabilidade com o teste-t de Student. Para o tempo de uso (Figura 6-a), percebe-sepredominância às $7 \mathrm{~h}$ e às 19 h. A fração de potência média é de 0,10 , enquanto varia de 0,06 a $0,16 \mathrm{com} 80 \%$ de confiabilidadeàs $7 \mathrm{~h}$. Para as $19 \mathrm{~h}$, a média também é de 0,10 , mas com variação de 0,03 a 0,16.

Uma forma de interpretar a Figura 6-a seria relacionála com o tempo de uso, ondeuma fração horária de 0,10 correspondea 6 minutos de uso na potênciominal, na respectiva hora do dia. Às $7 \mathrm{~h}$, por exemplo, o chuveiro é utilizado de uma fração de 0,063 a 0,167 (3,8 a 10 minutos na hora completa) e é utilizado de 193 a 590 Wh nesse horário, com 80\% de confiabilidade.

\section{Conclusões}

Este trabalho apresentou os usos finais de eletricidade e rotinas de uso dos equipamentos eletroeletrônicos por meio de auditoria energética em habitações de interesse social de Florianópolis-SC.

O maior uso final de eletricidade registrado é devido ao chuveiro elétrico, tanto para períodos de verão quanto de inverno, seguido dos refrigeradores, televisão e iluminação. No entanto, alguns autores apresentaram resultados diferentes, com refrigeradores respondendo pelos maiores usos finais, para a mesma região analisada. Este fato pode ser explicado pelas peculiaridades dessas habitações de interesse social, e pela amostra de tamanho reduzido.

O método apresentado se mostrou bastante útil para se determinar não somente os usos finais, mas as rotinas de uso associadas, de forma que seja possível uma posterior análise do desempenho térmico dessas habitações por simulação computacional.

Ao serem definidos usos finais para amostras pequenas, pode-se investir em programas governamentais mais específicos, capazes de gerar melhores resultados.

Estratégias de aquecimento solar de água, refrigeradores e televisões mais eficientes, seriam recomendadas alternativas para esse tipo de habitação, bem como a utilização de lâmpadas mais eficientes. No entanto, elas somente podem ser comprovadas com estudos técnicos mais específicos.

\section{Referências}

ALMEIDA, M. A.; SCHAEFFER, R.; LA ROVERE, E. L. The potential for electricity conservation and peak load reduction in the residential sector of Brazil. Energy, v. 26, n. 4, p. 413-429, 2001.

BIN, S.; DOWLATABADI, H. Consumer lifestyle approach to US energy use and the related $\mathrm{CO} 2$ emissions. Energy Policy, v. 33, n. 2, p. 197-208, 2005.

BRASIL. Pesquisa de orçamentos familiares 20022003 - Perfil das despesas no Brasil. Instituto Brasileiro de Geografia e Estatística (IBGE). Rio de Janeiro, 2007.

2011.

Decreto n. 7655, de 23 de Dezembro de

Balanço Energético Nacional 2012: Ano base 2011. Empresa de Pesquisa Energética (EPE). Rio de Janeiro, 2012a.

Programa Nacional de Conservação de Energia Elétrica. Resultados PROCEL 2012: ano base 2011. Eletrobrás, 2012b.

Programa de Eficiência Energética 2012 Enersul: Rede Energia. São Paulo, 2012c. Disponível em: < http://www.redenergia.com/enersul >, acesso em Março de 2013.

CHUNG-SHENG, Z.; SHU-WEN, N. I. U.; XIN, Z. Effects of household energy consumption on environment and its influence factors in rural and urban areas. Energy Procedia, v. 14, p. 1, 2012.

DRUCKMAN, A. AND JACKSON, T. The carbon footprint of UK households 1990-2004: A socioeconomically disaggregated, quasi-multi-regional input-output model. Ecological Economics, v. 68, v. 7, p. 2066-2077, 2009. 
FEDRIGO, N. S.; GHISI, E.; LAMBERTS, R. Usos finais de energia elétrica no setor residencial brasileiro. I Encontro Nacional e VI Encontro Latino Americano de Conforto no Ambiente Construído. Anais... Natal, RN, 2009.

GHISI, E.; GOSCH, S.; LAMBERTS, R. Electricity end-uses in the residential sector of Brazil. Energy Policy, v. 35, n. 8, p. 4107-4120, 2007.

GHISI, E.; WESTPHAL, F. S.; LAMBERTS, R. Determinação do consumo de eletricidade e da potência de equipamentos através de medidor portátil. IV Encontro Nacional de Conforto no Ambiente Construído. Anais... Salvador, BA, 1997.

JANNUZZI, G. M. AND SCHIPPER, L. The structure of electricity demand in the Brazilian household sector. Energy Policy, v. 19, n. 9, p. 879-891, 1991.

PREFEITURA MUNICIPAL DE FLORIANÓPOLIS PMF. Plano Municipal de Habitação de Interesse Social: Produto 08. Secretaria Municipal de Habitação e Saneamento Ambiental (SMHSA), Florianópolis, SC, jun. 2011. Disponível em: < http://www.pmf.sc. gov.br/ >, acesso em Outubro de 2012.

SANTANA, E. L. P.; HAYASHI, T. C. Perfil de consumo de energia em residências na cidade de Salvador. IV Encontro Nacional de Conforto no Ambiente Construído. Anais... Salvador, BA, 1997.

SIEGEL, S. Estatísticas Não Paramétrica para Ciências do Comportamento. Editora Bookman. Segunda edição, 2006. 